\title{
MASERS AS CHEMICAL PROBES OF STAR-FORMING REGIONS
}

\author{
KARL M. MENTEN \\ Harvard-Smithsonian Center for Astrophysics \\ 60 Garden Street, Cambridge MA 02138, USA
}

\begin{abstract}
Maser emission from the $\mathrm{OH}, \mathrm{H}_{2} \mathrm{O}$, and $\mathrm{CH}_{3} \mathrm{OH}$ molecules is found toward hundreds of molecular cloud cores with recent and on-going star formation. The often very high intensities of the observed emission imply substantial abundances of the maser molecules, which are difficult or impossible to explain with ion-molecule gas phase chemistry. We summarize relevant observations which show that $\mathrm{OH}$ and Class II $\mathrm{CH}_{3} \mathrm{OH}$ masers form in the envelopes of ultracompact HII regions in which the high abundances of these molecules are produced by evaporation of methanol and water from icy dust grain mantles. In contrast, $\mathrm{H}_{2} \mathrm{O}$ masers form in outflows from protostellar objects, where high $\mathrm{H}_{2} \mathrm{O}$ abundances are produced by endothermic neutral-neutral reactions in hot postshock gas. Finally, we briefly discuss Class $\mathrm{I} \mathrm{CH}_{3} \mathrm{OH}$ masers, which also arise in interstellar outflows.
\end{abstract}

\section{Introduction - masers in star-forming regions}

Astrophysical masers are found in the molecular envelopes of mass-losing red giant and supergiant stars, in interstellar molecular clouds, and in the circumnuclear regions of external galaxies (for extensive reviews, see Reid \& Moran 1988, Cohen 1989, Watson 1993, the monograph by Elitzur 1991, and the conference proceedings edited by Clegg \& Nedoluha 1993). Emission from the most widespread interstellar maser molecules, hydroxyl $(\mathrm{OH})$, water $\left(\mathrm{H}_{2} \mathrm{O}\right)$, and methanol $\left(\mathrm{CH}_{3} \mathrm{OH}\right)$, is observed toward hundreds of highmass star-forming regions; $\mathrm{H}_{2} \mathrm{O}$ masers are also found toward numerous regions in which only low-mass stars are forming. Maser emission from a number of other molecules $\left(\mathrm{CH}, \mathrm{SiO}, \mathrm{NH}_{3}\right.$, and $\left.\mathrm{H}_{2} \mathrm{CO}\right)$ is observed toward a few high-mass star-forming regions. Because of their very high brightness 
and compact size, masers can be observed with interferometric techniques at high spatial resolution. In particular, Very Long Baseline Interferometry (VLBI) yields milliarcsecond resolution, allowing mapping of the maser distributions on AU size scales and determination of three-dimensional velocities via proper motion measurements.

Observed maser intensities are in many cases strongly dependent on the geometry of the emitting regions and it is thus generally difficult to infer physical parameters of the emitting regions, such as density and kinetic temperature, from observations of a maser line with high accuracy. However, for many maser molecules, in particular $\mathrm{H}_{2} \mathrm{O}$ and $\mathrm{CH}_{3} \mathrm{OH}$, the increased observational accessibility of the (sub)millimeter wavelength range has led to the discovery of many different maser transitions. This allows multitransition studies, which, together with theoretical excitation calculations, lead to estimates of temperatures and densities in the masing regions (see, e.g., Neufeld \& Melnick 1991, $\left(\mathrm{H}_{2} \mathrm{O}\right)$; Cragg et al. $1992\left(\mathrm{CH}_{3} \mathrm{OH}\right)$; Cesaroni \& Walmsley $1991(\mathrm{OH})$ ). Table 1 summarizes the results of such studies; note that the quoted values for the molecular hydrogen density, $n\left(\mathrm{H}_{2}\right)$, and kinetic temperature $\left(T_{\text {kin }}\right)$, are only order-of-magnitude estimates.

TABLE 1. Widespread Interstellar Masers

\begin{tabular}{llll}
\hline Species & Occurence & $\begin{array}{l}n\left(\mathrm{H}_{2}\right) \\
\left(\mathrm{cm}^{-3}\right)\end{array}$ & $\begin{array}{l}T_{\text {kin }} \\
(\mathrm{K})\end{array}$ \\
\hline $\mathrm{H}_{2} \mathrm{O}$ & High-velocity outflows from young stellar objects & $10^{9}$ & 500 \\
$\mathrm{CH}_{3} \mathrm{OH}$ & Class I: High-velocity outflows and cloud-cloud collisions & $10^{5}$ & 100 \\
& Class II: Envelopes of ultracompact HII regions & $10^{7}$ & 150 \\
$\mathrm{OH}$ & same as Class II $\mathrm{CH}_{3} \mathrm{OH}$ masers & $10^{7}$ & 150 \\
\hline
\end{tabular}

Obviously, maser lines are probes of hot, dense regions. In many cases this is immediately clear from the energy levels involved, which, e.g., for known $\mathrm{H}_{2} \mathrm{O}$ maser lines are between 200 and $1800 \mathrm{~K}$ above the ground state. Moreover, virtually all model calculations predict that the often very high maser intensities observed for interstellar $\mathrm{H}_{2} \mathrm{O}, \mathrm{CH}_{3} \mathrm{OH}$, and $\mathrm{OH}$ masers require abundance ratios for the masing molecules relative to $\mathrm{H}_{2}$ that are significantly higher than values predicted by gas-phase chemical models for and observed in quiescent, cold, dark clouds. In this paper we shall give an overview over the high-temperature chemistry relevant for the formation of the maser molecules. 


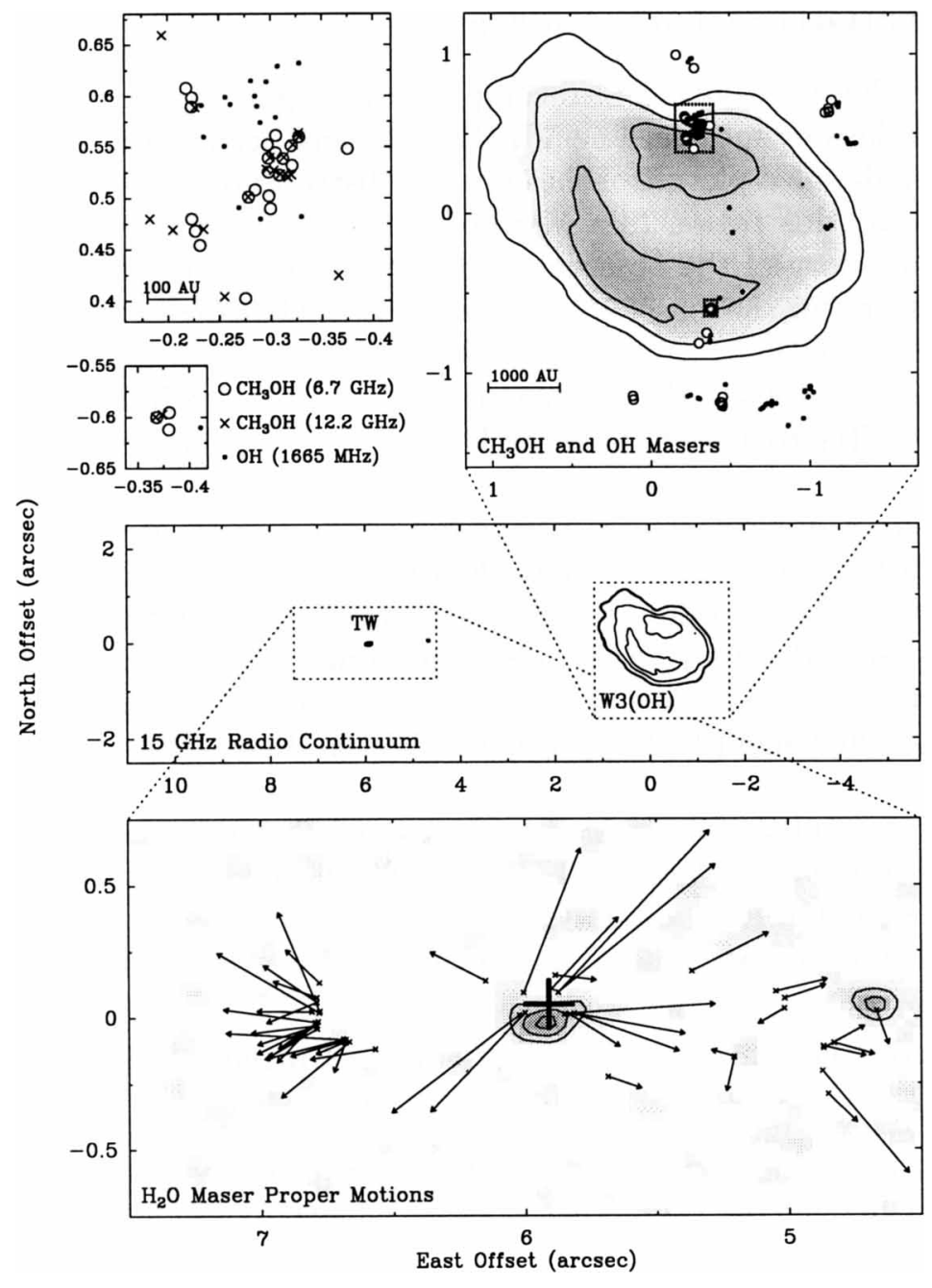

Figure 1. Distribution of maser emission in different molecular species in the neighbourhood of the ultracompact $\mathrm{HII}$ region $\mathrm{W} 3(\mathrm{OH})$, which dominates the $15 \mathrm{GHz}$ radio continuum map shown in the center panel. A blow-up is shown in the upper right panel with maser spots observed in the $1665 \mathrm{MHz} \mathrm{OH}$ and the $6.7 \mathrm{GHz} \mathrm{CH}_{3} \mathrm{OH}$ lines marked as black dots and open circles, respectively. Blow-ups of the two regions within the dotted rectangles are shown in the upper left panels, which also contain, as crosses, positions of maser spots identified in the $12 \mathrm{GHz} \mathrm{CH}_{3} \mathrm{OH}$ maser line (see Menten et al. 1992). Very weak non-thermal continuum emission, marked $\mathrm{TW}$, is seen $\approx 6^{\prime \prime}$ east of W3(OH); it is coincident with a compact dust emission core discovered by Turner \& Welch (1984). The lower panel, taken from Reid et al. (1995), shows $\mathrm{H}_{2} \mathrm{O}$ maser proper motion vectors measured by Alcolea et al. (1992) as arrows overlaid on the continuum map. The $\mathrm{H}_{2} \mathrm{O}$ center of expansion, marked by the cross, is coincident with the non-thermal radio source. 


\section{The $\mathrm{W} 3(\mathrm{OH})$ region as an example}

W3 $(\mathrm{OH})$ is the prototypical ultracompact HII region/maser source. Since the earliest days of spectral line VLBI, ever more accurate measurements have led to a detailed picture of the emission distributions of different maser species toward this region (see Moran 1994 for a historical review). Fig. 1 gives a summary of the observational picture. Interferometrically determined positions for the masers observed in the $22 \mathrm{GHz} 6_{16} \rightarrow 5_{23}$ transition of $\mathrm{H}_{2} \mathrm{O}$, the $6.7 \mathrm{GHz} 5_{1} \rightarrow 6_{0} A^{+}$and the $12 \mathrm{GHz} 2_{0} \rightarrow 3_{-1} E$ transitions of $\mathrm{CH}_{3} \mathrm{OH}$ and the $1665 \mathrm{MHz} \mathrm{OH}$ hyperfine transition are indicated. All of the $\mathrm{OH}$ and $\mathrm{CH}_{3} \mathrm{OH}$ maser spots are found toward the western half of the ultracompact HII region and on scales of 100 AU or less their emission regions overlap. Moreover, where masers from both species are found in the same area, they have similar radial velocities (Menten et al. 1992).

In contrast, $\mathrm{H}_{2} \mathrm{O}$ masers are not observed toward the ultracompact $\mathrm{HII}$ region, but toward a very weak radio continuum source about $6^{\prime \prime}$ or 0.06 pc east of it that is coincident with a hot, compact dust and molecular line emission core first identified by Turner \& Welch (1984). VLBI proper motion studies have shown that the $\mathrm{H}_{2} \mathrm{O}$ masers form a well-defined bipolar outflow whose center of expansion is coincident with the radio continuum source (Alcolea et al. 1992). Reid et al. (1995) have shown that the radio continuum emission from the latter source is non-thermal and may represent a jet originating from the young stellar object driving the $\mathrm{H}_{2} \mathrm{O}$ outflow.

\section{3. $\mathrm{OH}$ and $\mathrm{CH}_{3} \mathrm{OH}$ masers around ultracompact $\mathrm{HII}$ regions}

Elitzur \& de Jong (1978) first proposed that $\mathrm{OH}$ maser emission from ultracompact HII regions such as $\mathrm{W} 3(\mathrm{OH})$ arises from the compressed and heated shell behind the shock driven into ambient molecular cloud material by the expansion of the HII region. General support for this scenario comes from VLBI $\mathrm{OH}$ proper motion studies, which suggest that the $\mathrm{OH}$ maser shell around $\mathrm{W} 3(\mathrm{OH})$ is expanding with a velocity of a few $\mathrm{km} \mathrm{s}^{-1}$ (Bloemhof et al. 1992). The $\mathrm{OH}$ to $\mathrm{H}_{2}$ abundance ratio, $\left[\mathrm{OH} / \mathrm{H}_{2}\right]$, in the masing region has been estimated as several times $10^{-7}$ (Wilson et al. 1990); detailed model calculations using this value, $\mathrm{H}_{2}$ densities of a few times times $10^{7} \mathrm{~cm}^{-3}$, and temperatures around $150 \mathrm{~K}$ successfully reproduce the observed pattern of maser emission in certain $\mathrm{OH}$ lines and absorption in others (Cesaroni \& Walmsley 1991).

In the Elitzur \& de Jong picture, the $\mathrm{OH}$ molecules are produced by photodissociation of $\mathrm{H}_{2} \mathrm{O}$ that is abundantly produced in the hot postshock gas. An alternative $\mathrm{H}_{2} \mathrm{O} / \mathrm{OH}$ production scheme, presented by Hartquist et al. (1995) was motivated by the observed coexistence of $\mathrm{OH}$ and $\mathrm{CH}_{3} \mathrm{OH}$ masers in W3(OH) (see §2) and the evidence for substantial methanol abun- 
dances implied by the very strong masers observed in the latter species and, more quantitatively, by observations of quasi-thermal methanol lines in absorption against the radio continuum emission of the ultracompact HII region. The observations show that the absorption covers only the western half of the HII region, toward which the masers are observed (Wilson et al. 1991) and imply very high methanol abundance ratios $\left[\mathrm{CH}_{3} \mathrm{OH} / \mathrm{H}_{2}\right]$ $>10^{-6}$ (Menten et al. 1985, 1986). Hartquist et al. (1995) examined possible gas-phase production pathways for methanol and concluded that they are incapable of producing the observed $\mathrm{CH}_{3} \mathrm{OH}$ abundances under the conditions in the masing region. They propose that large quantities of $\mathrm{CH}_{3} \mathrm{OH}$ (and $\mathrm{H}_{2} \mathrm{O}$ ) are injected into the gas phase by evaporation off icy dust grain mantles, where methanol may have formed by hydrogenation of $\mathrm{CO}$ accreted from the gas phase (see, e.g. Charnley et al. 1992). Evaporation sets in at temperatures $\gtrsim 100 \mathrm{~K}$, which are easily reached in the relatively weak shock driven by the expanding HII region.

Direct evidence for the above scenario is provided by infrared spectroscopy, which has established methanol ice as a significant constituent of dust grain mantles by ascribing absorption features at various wavelengths to different vibrational bands of solid $\mathrm{CH}_{3} \mathrm{OH}$ (e.g., Grim et al. 1991; Allamandola et al. 1992; Skinner et al. 1992). The inferred solid methanol abundances are substantial, ranging up to 50 percent of the abundance of solid $\mathrm{H}_{2} \mathrm{O}$, which is thought to be the dominant ice component. Several of the sources with strong ice features, such as Mon R2, W33A, and AFGL 2136, also show powerful $\mathrm{OH} / \mathrm{CH}_{3} \mathrm{OH}$ maser action. Unfortunately, such infrared absorption data do not exist for some of the sources that are very well-studied in the radio region, such as $\mathrm{W} 3(\mathrm{OH})$. Most ultracompact HII regions have very massive, warm envelopes that, while emitting copiously at far-infrared wavelengths, strongly attenuate the near-infrared emission from the hot, inner regions, making absorption spectroscopy difficult.

Some of the $\mathrm{H}_{2} \mathrm{O}$ injected into the gas phase is photodissociated into $\mathrm{OH}$. Since $\mathrm{H}_{2} \mathrm{O}, \mathrm{OH}$, and $\mathrm{CH}_{3} \mathrm{OH}$ have similar photodissociation rates, all three of these molecules may coexist with substantial abundances in the same region of the envelope, which has a density $\left(\approx 10^{7} \mathrm{~cm}^{-3}\right)$ and temperature $(150-200 \mathrm{~K})$. These conditions are conducive to the formation of $\mathrm{OH}$ and Class II $\mathrm{CH}_{3} \mathrm{OH}$ masers, which are most likely pumped by farinfrared radiation via excitation of higher rotational levels in the case of $\mathrm{OH}$ (e.g., Cesaroni \& Walmsley 1991) and levels in the first torsionally excited state for $\mathrm{CH}_{3} \mathrm{OH}$ (Menten 1991). In contrast, despite the substantial $\mathrm{H}_{2} \mathrm{O}$ abundance, the physical conditions in this region do not produce $\mathrm{H}_{2} \mathrm{O}$ maser action (at least not in the most-studied $22 \mathrm{GHz}$ line), which requires substantially higher densities and temperatures (see next section). However, recent infrared spectroscopy of vibrational lines of $\mathrm{H}_{2} \mathrm{O}$ with ISO 
has led to the direct detection of abundant hot water in the gas-phase toward one $\mathrm{OH} / \mathrm{Class}$ II $\mathrm{CH}_{3} \mathrm{OH}$ maser source, AFGL 2136 (van Dishoeck \& Helmich 1996). For ultracompact HII regions that have high enough nearinfrared flux densities to allow IR spectroscopical studies, high resolution radio (maser and absorption) and infrared absorption data probe the same line-of-sight and complementary studies in both wavelength regimes have great potential for studying the physical conditions and chemistry around newly formed massive stars.

\section{Water masers as tracers of shocked regions}

Water masers in star-forming regions often have spectra with large radial velocity spreads, which in extreme cases are of order several hundred $\mathrm{km} \mathrm{s}^{-1}$. This indicates that these masers form in outflows from young stellar objects, a notion that has been confirmed by VLBI proper motion studies (e.g., Genzel et al. 1979; Gwinn et al. 1992; Alcolea et al. 1992, see Fig. 1). Shocks are obvious consequences of the observed supersonic motions and are capable of providing the maser pump energy via collisional excitation of the $\mathrm{H}_{2} \mathrm{O}$ molecules, which are abundantly produced by neutral-neutral reactions proceeding at the high temperatures of the postshock gas. The maser emission arises from velocity-coherent filaments in the post-shock material.

A model for $\mathrm{H}_{2} \mathrm{O}$ masers based on this scenario was developed by Elitzur et al. (1989). In particular, they consider the case of a high velocity $(\geq 50$ $\mathrm{km} \mathrm{s}^{-1}$ ) $J$-shock in which the molecules are dissociated. Their calculations indicate that a substantial column (a few times $10^{22} \mathrm{~cm}^{-2}$ ) of the postshock gas is at a kinetic temperature around $400 \mathrm{~K}$, which results from the heat of $\mathrm{H}_{2}$ reformation on dust grains. At this temperature, the chemistry results in a large $\mathrm{H}_{2} \mathrm{O}$ abundance $\left(\left[\mathrm{H}_{2} \mathrm{O} / \mathrm{H}_{2}\right] \sim 10^{-4}\right)$ and the masers can be efficiently pumped (Neufeld \& Melnick 1991).

In the past, direct observational information on the temperature of the masing regions was difficult to obtain, because only a single $\mathrm{H}_{2} \mathrm{O}$ maser line, the $22 \mathrm{GHz}$ transition, could be observed. Recently, various other $\mathrm{H}_{2} \mathrm{O}$ maser transitions have been detected at (sub)millimeter wavelengths (Menten et al. 1990a,b; Cernicharo et al. 1990; Melnick et al. 1993). Melnick et al. use contemporaneously measured spectra of several submillimeter lines to derive intensity ratios which they compare with the values predicted from model calculations. They find that for all of the six star-forming regions they studied, the kinetic temperature has to be in excess of $900 \mathrm{~K}$ to explain the observed line ratios. This suggests that the masers are formed in the post-shock gas behind non-dissociative $C$-shocks, which can have higher temperatures than gas behind $J$-shocks. 
To realize the full potential of these multi-transition studies requires high resolution observations of all the maser lines. The available submillimeter data were taken with beamwidths of $\approx 20^{\prime \prime}$ and significant uncertainties on the small scale structure at present preclude more than qualitative conclusions. Future interferometers such as the Submillimeter Array, currently under construction by the Smithsonian Astrophysical Observatory (Moran \& Ho 1994), or the Millimeter Array, planned by the National Radio Observatory, will allow (sub)millimeter wavelength observations at resolutions comparable to those currently obtainable with the Very Large Array (VLA) for the $22 \mathrm{GHz}$ line.

\section{Class I methanol masers and molecular outflows}

Class I $\mathrm{CH}_{3} \mathrm{OH}$ maser sources show maser action in a number of transitions in which Class II $\mathrm{CH}_{3} \mathrm{OH}$ maser sources are not masing and vice versa (see Menten 1991). Provided that all other conditions (high $\mathrm{CH}_{3} \mathrm{OH}$ abundance!) are conducive to methanol maser formation, the absence or presence of strong far-infrared continuum emission seems to be the factor deciding whether Class I or Class II masers, respectively, are produced (Menten 1991; Cragg et al. 1992). In particular, inversion of Class I methanol maser transitions over a wide range of physical conditions by collisionial excitation is expected from basic properties of the methanol molecule and verified by statistical equilibrium calculations (Menten 1991; Johnston et al. 1991; Cragg et al. 1992). This explains why Class I methanol masers, although usually found in the general vicinity of newly formed high-mass stars, are frequently offset by up to $1 \mathrm{pc}$ from the nearest compact HII regions or strong infrared sources, in contrast to Class II masers, which need the strong circumstellar far-infrared radiation for their excitation and, therefore, only arise from the immediate neighbourhood of young stellar objects (see sections 2 and 3 above).

Almost certainly, substantial $\mathrm{CH}_{3} \mathrm{OH}$ abundances are necessary to produce observable Class I masers which, as in the case of Class II masers, might be produced by evaporation of $\mathrm{CH}_{3} \mathrm{OH}$ off icy dust grain mantles by shock heating. Moreover, given the high velocities of some of these outflows post-shock temperatures of several thousand $\mathrm{K}$ can be reached. At such temperatures endothermal neutral-neutral reactions are capable of producing very large $\mathrm{CH}_{3} \mathrm{OH}$ abundances (Hartquist et al. 1995).

Direct observational evidence for a relationship of Class I methanol masers and outflows producing the shocks has been presented by Plambeck \& Menten (1990), who mapped the $95 \mathrm{GHz} 8_{0} \rightarrow 7_{1} A^{+}$Class I methanol maser transition and, simultaneously, the $J=2 \rightarrow 1$ transition of CS toward the DR 21 star-forming region. As shown in Fig. 2, in this region a 


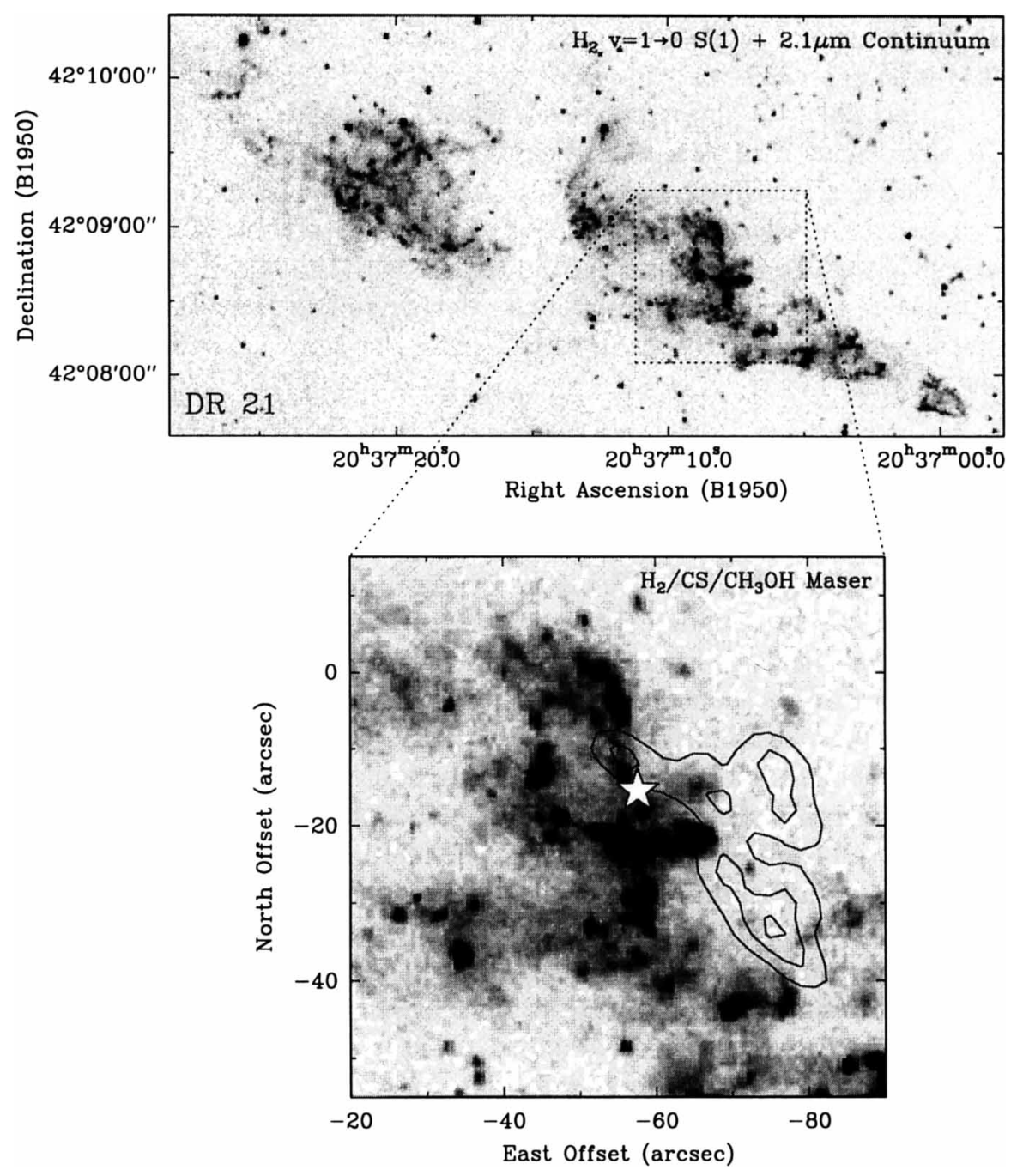

Figure 2. The upper panel shows a narrow-band $2.122 \mu \mathrm{m}$ infrared image of the DR 21 region (from Davis \& Smith 1996). The extended diffuse emission is from the $\mathrm{S}(1)$ line of vibrationally excited $(v=1 \rightarrow 0) \mathrm{H}_{2}$. No continuum has been subtracted from the image. Several ultracompact $\mathrm{HII}$ regions are located within the dark dust lane separating the $\mathrm{H}_{2}$ emission into two lobes. A blow-up of the region within the dotted square is displayed in the lower panel, which, in addition to the infrared emission, also shows emission in the $J=2 \rightarrow 1$ transition of CS (contour lines) and the location of a maser in the $8_{0} \rightarrow 7_{1} A^{+}$ transition of $\mathrm{CH}_{3} \mathrm{OH}$ (star symbol). The CS and $\mathrm{CH}_{3} \mathrm{OH}$ data were obtained with the BIMA interferometer by Plambeck \& Menten (1990).

giant bipolar flow several parsecs across is traced by vibrationally excited molecular hydrogen (see, e.g. Davis \& Smith 1996). The methanol maser is located between the outer edge of the shocked region and a CS emission 
core, which most likely traces dense, ambient gas the outflow is impinging on. Recently, Liechti \& Walmsley (1996) have also mapped thermal methanol emission arising from this interaction region.

Because of their relatively high intensities, Class I methanol masers are signposts for interaction zones of outflows with ambient material (e.g., Orion-KL, Johnston et al. 1991) and/or, as in the case of Sgr B2, for cloudcloud collisons (Mehringer \& Menten 1996).

\section{Acknowledgements}

I would like to thank Chris Davis for making the DR 21 infrared image used in Fig. 2 available in computer-readable form.

\section{References}

Alcolea, J., Menten, K. M., Moran, J. M., Reid, M. J. 1992, in Astrophysical Masers, ed. A. W. Clegg \& G. E. Nedoluha (Springer, Berlin), p. 225

Allamandola, L. J., Sandford, S. A., Tielens, A. G. G. M., Herbst, T. M. 1992, ApJ, 399, 134

Bloemhof, E. E., Reid, M. J., Moran, J. M. 1992, ApJ, 397, 500

Cernicharo, J., Thum, C., Hein, H., John, D., Garcia, P., Mattioco, F. 1990, A\&A, 231, L15

Cesaroni, R., Walmsley, C. M. 1991, A\&A, 241, 537

Charnley, S. B., Tielens, A. G. G. M., Millar, T. J., 1992, ApJ, 399, L71

Clegg, A. W., Nedoluha, G. E., eds. 1993, Astrophysical Masers (Springer, Berlin)

Cohen, R. J. 1989, Rep. Prog. Phys., 52, 881

Cragg, D. M., Johns, K. P., Godfrey, P. D., Brown, R. D. 1992, MNRAS, 259, 203

Davis, C. J., Smith, M. D. 1996, A\&A, 310, 961

Elitzur, M. 1991, Astronomical Masers, (Kluwer, Dordrecht)

Elitzur, M., de Jong, T. 1978, A\&A, 67, 323

Elitzur, M., Hollenbach, D. J., McKee, C. F. 1989, ApJ, 346, 983

Genzel, R., Reid, M. J., Moran, J. M., Downes, D. 1979, ApJ, 244, 884

Grim, R. J. A., Baas, F., Geballe, T. R., Greenberg, J. M., Schutte, W. 1991, A\&A, 243, 473

Gwinn, C. R., Moran, J. M., Reid, M. J. 1992, ApJ, 393, 149

Hartquist, T. W., Menten, K. M., Lepp, S., Dalgarno, A. 1995, MNRAS, 272, 184

Johnston, K. J., Gaume, R., Stolovy, S., Wilson, T. L., Walmsley, C. M., Menten, K. M. 1992, ApJ, 385, 232

Liechti, S., Walmsley, C. M. 1996, A\&A, in press

Mehringer, D. M., Menten, K. M. 1996, ApJ 474, 346

Melnick, G. J., Menten, K. M., Phillips, T. G., Hunter, T. 1993, ApJ, 416, L37

Menten, K. M. 1991, in Skylines (Proc. Third Haystack Observatory Meeting), ed. A. D. Haschick \& P. T. P. Ho (ASP, San Francisco), p. 119

Menten, K. M., Johnston, K. J., Wilson, T. L., Walmsley, C. M., Mauersberger, R., Henkel, C. 1985, ApJ, 293, L83

Menten, K. M., Melnick, G. J., Phillips, T. G. 1990a, ApJ, 350, L41

Menten, K. M., Melnick, G. J., Phillips, T. G., Neufeld, D. A. 1990b, ApJ, 363, L27

Menten, K. M., Reid, M. J., Pratap, P., Moran, J. M., Wilson, T. L. 1992, ApJ, 401, L39

Menten, K. M., Walmsley, C. M., Henkel, C., Wilson, T. L. 1986, A\&A, 157, 318

Moran, J. M. 1994, in The Structure and Content of Molecular Clouds, ed. T. L. Wilson \& K. J. Johnston (Springer, Berlin), p. 89 
Moran, J. M., Ho, P. T. P. 1994, Proc. SPIE, 2200, Amplitude and Intensity Spatial Interferometry II, ed. J. B. Breckinridge, p. 335

Neufeld, D. A., Melnick, G. J. 1991, ApJ, 368, 215

Plambeck, R. L., Menten, K. M. 1990, ApJ, 364, 555

Reid, M. J., Argon, A. L., Masson, C. R., Menten, K. M., Moran, J. M. 1995, ApJ, 443, 238

Reid, M. J., Moran, J. M. 1988, in Galactic and Extragalactic Radio Astronomy, eds. G. Verschuur \& K. I. Kellermann (Springer, Berlin), p. 225

Skinner, C. J., Tielens, A. G. G. M., Barlow, M. J., Justtanont, K. 1992, ApJ, 399, L79

Turner, J. L., Welch, W. J. 1984, ApJ, 287, L81

van Dishoeck, E. F., Helmich, F. P. 1996, A\&A, 315, ***

Watson, W. D. 1993, in The Structure and Content of Molecular Clouds, eds. T. L. Wilson \& K. J. Johnston (Springer, Berlin), p. 109

Wilson, T. L., Johnston, K. J., Mauersberger, R. 1991, A\&A, 251, 220

Wilson, T. L., Walmsley, C. M., Baudry, A. 1990, A\&A, 231, 159

\section{Discussion}

Schutte: Is there a possibility to observe deuterated masers (e.g. $\mathrm{CH}_{2} \mathrm{DOH}$ which has an abundance $4 \%$ of that of methanol in hot cores)?

Menten: In some cases, e.g. $\mathrm{CH}_{2} \mathrm{DOH}, \mathrm{HDO}, \mathrm{NH}_{2} \mathrm{D}$, the energy level structure of the deuterated species is quite different from that of the main isotope. Since for many maser lines the mechanism depends on details of the energy level arrangement, one does not necessarily expect maser action in deuterated isotopes even if some transitions of the main isotope are masing. Possible maser lines from deuterated species would generally be quite weak because of the relatively low abundances of the deuterated isotopomers. Note that weak maser emission has been observed from other rare isotopomers, such as ${ }^{15} \mathrm{NH}_{3}$.

Mundy: Bruce Wilking, Al Wootten, and collaborators have surveyed low-mass young stellar objects and find that most have $\mathrm{H}_{2} \mathrm{O}$ masers, which are time variable. Nearly all of these masers are within $100 \mathrm{AU}$ of the central star. Is that result compatible with your view that the $\mathrm{H}_{2} \mathrm{O}$ masers arise in outflows?

Menten: Certainly VLBI proper motion studies provide very good evidence that $\mathrm{H}_{2} \mathrm{O}$ masers in high-mass star-forming regions arise in outflows. For low-mass starforming regions such proper motion studies are not available yet. Interestingly, one would expect the conditions in circumstellar disks at, say, a $100 \mathrm{AU}$ from the central star also to be conducive to water maser action. However, VLA observations of sources that showed triple-peaked spectra, strongly suggesting emission from a disk with Keplerian rotation (see Cesaroni, R. 1990, A\&A 233, 513), failed to produce evidence for the disk scenario.

Slysh: With what kind of sources are Class I methanol masers associated?

Menten: These masers are found in high-mass star-forming regions and arise in the interaction regions between bipolar outflows and dense ambient material or in cloud-cloud collisions. 